\title{
Understanding the Impact of the PC5 Resource Grid Design on the Capacity and Efficiency of LTE-V2X in Vehicular Networks
}

\author{
Leandro Miguel Lopez, ${ }^{1}$ Charmae Franchesca Mendoza, ${ }^{1}$ Jordi Casademont $\mathbb{D}^{1},{ }^{1,2}$ \\ and Daniel Camps-Mur ${ }^{2}$ \\ ${ }^{1}$ Universitat Politècnica de Catalunya, Barcelona 08034, Spain \\ ${ }^{2}$ i2CAT Foundation, Barcelona 08034, Spain \\ Correspondence should be addressed to Jordi Casademont; jordi.casademont@upc.edu
}

Received 3 December 2019; Accepted 24 April 2020; Published 1 June 2020

Academic Editor: Rüdiger C. Pryss

Copyright (C) 2020 Leandro Miguel Lopez et al. This is an open access article distributed under the Creative Commons Attribution License, which permits unrestricted use, distribution, and reproduction in any medium, provided the original work is properly cited.

\begin{abstract}
Vehicular communications will foster mobility services and enable mass adoption of future autonomous vehicles, interchanging huge amount of data acquired from vehicles' sensors. 3GPP Release 14 presents the first standard for supporting V2X in LTE. Several enhancements are introduced, including a new arrangement of the physical resource grid, where subchannels are the minimum resource unit instead of Resource Blocks. The resource grid is defined by several design parameters, some of them with constraints imposed by 3GPP specifications, that affect the maximum message transmission rate and efficiency of the system. Moreover, the optimum choice of these parameters is closely linked to message length, which is another variable parameter. This paper provides an analysis of the relationship between these design parameters (Resource Block per Subchannel, Transport Block Size Index, and coding rate), message size, and the system's maximum capacity and efficiency. In doing so, we do not consider channel reuse or radio transmission characteristics because the focus of this paper is trying to find the resource grid design parameters that optimize system capacity, which is a very important aspect to consider by V2X operators.
\end{abstract}

\section{Introduction}

The ecosystem of vehicular networks opens a wide horizon of new opportunities in different sectors as in businesses and application development, which include artificial intelligence algorithms, communication protocol integration, and new wireless technologies to communicate with vehicles, pedestrians, road infrastructure, and management centres. Nowadays, there is a myriad of concepts, different protocol architectures, and operational functionalities which need to be coordinated and integrated. Reference [1] explains in detail all these concepts.

Standardization organizations and industry began to move several years ago to define the first mobility services, also known as Intelligent Transport Systems (ITS), which are based on periodic exchange of data packets that convey status information to neighbouring vehicles. The basic cases are, for Europe, the Cooperative Awareness Message (CAM) defined by the European Telecommunications Standards Institute (ETSI) and, for the United States of America (USA), the Basic Safety Message (BSM) defined by the Society of Automotive Engineers (SAE), both of them transmitted with a periodicity of $1-10 \mathrm{~Hz}$, which report the position, speed, and direction of a vehicle. Both organizations have standardized several more messages, and some others are nowadays under development, as, for instance, the so-called Collective Perception Messages (CPM) where vehicles will interchange information acquired with their onboard sensors, such as objects in the road, their dimensions, presence of pedestrians, and distances between the object and the transmitting vehicle. It is for this reason that the communication system will have to deal with a high variability of messages with different payload lengths. Nevertheless, some of them will be transmitted much more often than others. 
Up to now, IEEE 802.11p has been the de facto wireless technology standard for Vehicle-to-Everything (V2X) communications. It is a relatively mature technology and has already been validated by over a decade of field trials. In spite of that, IEEE 802.11p, which uses Carrier-Sense Multiple Access with Collision Avoidance (CSMA/CA), suffers from a high level of collisions under heavy traffic conditions, mainly due to hidden terminal situations. Moreover, there is also a lack of clear plans for future enhancements and a lack of business models to support the deployment of additional infrastructure such as Road Side Units (RSUs) [2].

Long-Term Evolution- (LTE-) based V2X from the 3rd Generation Partnership Project (3GPP) is a relatively new alternative to IEEE $802.11 \mathrm{p}$-based V2X communications. The first version of LTE-V2X was published in June 2017 under Release 14, which came with numerous enhancements to the existing Device-to-Device (D2D) communications in order to accommodate vehicular communications. The proposed enhancements include a new arrangement of the resource grid of the physical layer and two types of D2D channel access mechanisms: (i) a mechanism coordinated by the evolved NodeB (eNB), named Mode 3, and (ii) a distributed mechanism, where User Equipment (UE) accesses the channel on its own, named Mode 4. Moreover, LTEV2X employs different radio interfaces: (i) an interface between the vehicle and eNB, named LTE-Uu, and (ii) an interface between vehicles, named PC5.

Current specifications of 3GPP state that PC5 communication takes place in one channel inside the unlicensed frequency band $5855 \mathrm{MHz}-5925 \mathrm{MHz}$ named ITS-G5, while LTE-Uu uses part of the licensed spectrum assigned to the operator that owns the eNB. Therefore, as the resource grid is fully dedicated to PC5 communication (i.e., any communication between vehicles and eNBs takes place over the operator-licensed spectrum), our analysis applies to both Modes 3 and 4 and uncovers a high sensitivity between the application message size and the aforementioned design parameters.

One of the main problems to operate a PC5 resource grid is that the organization in charge of its administration, which can be the regulator or a telecommunication operator, has to select the value of several design parameters such as the Resource Block per Subchannel, Transport Block Size Index, and coding rate. In this paper, we study how the selection of these values, which is intrinsically linked to the size of the transmitted messages and has several constraints imposed by the 3GPP specifications, affects the efficiency of the system and the number of messages per second that the system will be able to transmit in a specific geographical area. Our main contribution in this paper is the analysis of the theoretical maximum message transmission capacity and efficiency of the PC5 resource grid and the variation that they suffer when ITS services change the payload of their messages. In our analysis, we do not consider channel reuse or interference effects, as our goal is to study the operational upper bounds under the influence of these design parameters. This study has direct application for those technicians in charge of the PC5 network setup as this analysis provides a reference starting point of the expected maximum performance of the system.
Up to now, the performance of the PC5 channel has been intensely analysed from different points of view, mainly taking into account channel reuse and interference effects. Without having to provide an extended review of these works, we can find some examples of these studies, for instance $[3,4]$, which present a general overview of the LTE-V2X and provide simulation results under realistic traffic conditions for the behaviour of the sensing-based Semi-Persistent Scheduling (SPS) Medium Access Control (MAC) algorithm for channel reuse in terms of Packet Delivery Ratio (PDR). [5] analyses the impact of PHY and MAC design parameters such as the sensing period, power threshold, time window, or time before evaluation, on the system performance in terms of the Packet Reception Ratio (PRR) and update delay. [6] presents a comparative of congestion control mechanisms for vehicular networks and proposes a new one. [7] is a 3GPP technical report that presents the suggested evaluation methodology to study LTEV2X and provide many simulation results of PRR under multiple scenarios with channel reuse. [8] shows an innovative approach proposing a new metric called Spatial Capacity, which provides the total amount of bits that can be successfully delivered in $1 \mathrm{~km}$ and $1 \mathrm{~s}$.

However, our work eschews these concepts in order to focus on the upper bounds of the system capacity which are affected by the resource grid design parameters. To the best of our knowledge, this is the first paper that studies how the capacity and efficiency of the PC5 resource grid is affected by (i) the number of Resource Blocks per Subchannel, (ii) the Transport Block Size Index and coding rate, (iii) the type of V2X message and the associated traffic model (derived from a real ETSI ITS communication protocol stack), and (iv) the transmission time interval and message size. Reference [9] provides an interesting work where the authors relate the Modulation and Coding Scheme (MCS), which can be mapped to the Transport Block Size Index, with two parameters, firstly, with the awareness and reuse ranges, and then they derive the maximum number of neighbour cars that the system can sustain. Our work differs from theirs in two approaches. As reference [9] considers channel reuse, their system's maximum capacity computations are affected not only by resource grid design parameters but also by the interference produced by this channel reuse. Moreover, they focus on MCS but do not deal with subchannel design (number of Resource Blocks per Subchannel), which affects the system performance when there is a transmission of massages of different sizes, which happens in most real use cases, or the effect of the 3 GPP specification restrictions when the message size varies.

This paper is organized as follows. Section 2 provides an overview of the LTE-V2X resource grid structure. Section 3 proposes a mathematical procedure to solve the problem of choosing the resource grid design parameters using the most common message length. Section 4 introduces traffic models and message types, commonly used by V2X applications. Sections 5 and 6 derive, respectively, the maximum capacity and efficiency achieved by the PC5 resource grid when messages do not have the most common message length. Finally, Section 7 summarizes and concludes the paper. 


\section{The LTE-V2X Resource Grid Structure}

Release 12 D2D was not designed with the strict latency and vehicle density requirements of $\mathrm{V} 2 \mathrm{X}$ in mind. In order to improve the support for V2X, Release 14 introduced a new arrangement on the resource grid. Figure 1 illustrates how messages fit in the new structure that keeps repeating over time. As vehicles need to send messages periodically (usually every $100 \mathrm{~ms}$ ), the transmission pattern keeps also repeating with the same periodicity, as illustrated in Figure 2.

In the time domain, the resource grid is divided into subframes of $1 \mathrm{~ms}$ each. In the frequency domain, the grid is divided, in every subframe, into physical Resource Blocks (RBs) that span $180 \mathrm{kHz}$ each with 12 subcarriers of $15 \mathrm{kHz}$. Every subcarrier transmits a total of 14 symbols per subframe, in which 9 symbols are devoted to data transmission, 4 symbols are used for Demodulation Reference Signals (DMRS), and the remaining symbol is used for $\mathrm{Tx}-\mathrm{Rx}$ turnaround.

Apart from these fixed parameters, there are some others that depend on design implementation, and its selection will define the maximum system capacity and efficiency, as described below.

The number of RBs per subframe ( $\left.N_{\text {RB_SFR }}\right)$ depends on the available channel bandwidth. LTE-V2X supports channel bandwidths of $10 \mathrm{MHz}$ and $20 \mathrm{MHz}$, which would have 50 RBs per subframe and 100 RBs per subframe, respectively [10]. LTE-V2X supports QPSK and 16QAM modulation schemes. For QPSK, 2 bits per symbol are transmitted, while 4 bits per symbol are utilized in 16QAM. Since 9 symbols per $\mathrm{RB}$ are used for data transmission, this translates to 216 total bits per RB for QPSK and 432 bits for 16QAM. This total number of bits includes the payload plus redundancy to cope with transmission errors. Depending on the required transmission robustness, different coding rates (ratio between payload bits plus Cyclic Redundancy Check (CRC) and total transmitted bits) can be set.

RBs in the same subframe are grouped into subchannels. A key characteristic of LTE-V2X is that resource allocation is in subchannel granularity (rather than in RB granularity). Every subchannel is composed of control information and user data. The control portion occupies 2 RBs, corresponding to the Sidelink Control Information (SCI) transmitted in PSCCH (Physical Sidelink Control Channel). The SCI includes information such as the MCS (Modulation and Coding Scheme) and the resource reservation information needed in sensing-based semipersistent scheduling [11]. The group of RBs carrying user data in a subchannel is named the Transport Block (TB). A transmission of a TB requires the transmission of the corresponding $\mathrm{SCI}$, as the latter is necessary for the correct reception and demodulation of the TB. A TB is transmitted in the PSSCH (Physical Sidelink Shared Channel) and occupies a variable number of $\mathrm{RBs}$ depending on the message size. The PSCCH and PSSCH are either adjacent or nonadjacent to each other on the same subframe. Depending on this configuration, the allowed subchannel size or number of RBs per subchannel differs as specified in [12]. In the case of adjacent PSCCH-PSSCH, 5, $6,10,15,20,25,50,75$, or 100 RBs may be configured per

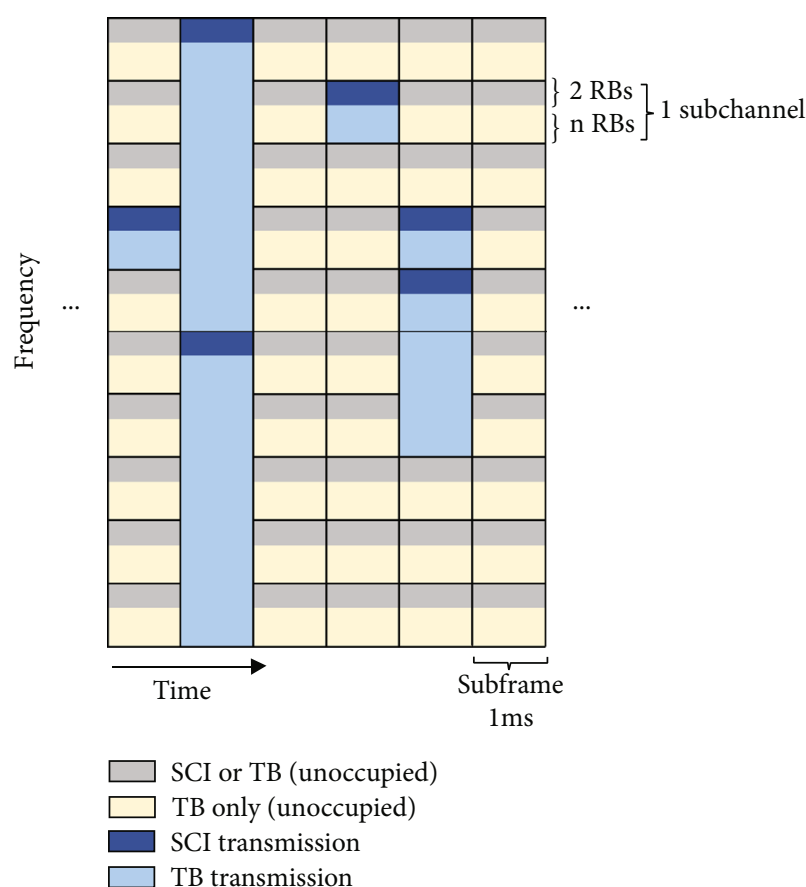

Figure 1: Structure of the PC5 resource grid with 10 subchannels per subframe.

subchannel, while $4,5,6,8,9,10,12,15,16,18,20,30,48$, 72 , or $96 \mathrm{RBs}$ per subchannel for the nonadjacent case. In this paper, only the adjacent case is considered. If the amount of data is large, it may occupy the succeeding subchannels (including the 2 RBs for the next SCI). Since the $\mathrm{PSCCH}$ and the PSSCH are always in the same subframe, it is possible to decode a PSSCH immediately after a PSCCH is recovered. Hence, the proposed vertical arrangement of data in the resource grid reduces decoding latency, which is one of the key objectives in V2X.

Each RB can be modulated using QPSK (modulation order 2) or 16QAM (modulation order 4) and different MCS Indexes $\left(I_{\mathrm{MCS}}\right)$. These two parameters are merged into a single one named the Transport Block Size Index $\left(I_{\mathrm{TBS}}\right)$ as indicated in Table 8.6.1-1 of [13]. $I_{\mathrm{TBS}}$ defines the robustness of the radio modulation and the coding rate, which in turn, affects the coverage area of radio transmissions and the number of bits that can be transmitted in a fixed-size TB. The lower the $I_{\mathrm{TBS}}$, the more robust the communication is and, therefore, the farther messages are correctly received, but the fewer the payload bits per fixed-size TB transmitted. Reference [9] provides values of typical distance ranges that can be reached with different MCS values.

The other relevant parameter is the coding rate which is calculated as [10]

$$
\text { Coding rate }=\frac{N_{\text {BITS_TB }}+24\left(N_{\mathrm{CB}}+1\right)}{N_{\text {BITS_SCH }}},
$$

where the TB payload size in bits $\left(N_{\text {BITS_TB }}\right)$ must be a valid TB size from Table 7.1.7.2.1-1 of [13], which specifies the corresponding $\mathrm{TB}$ size in bits given the $I_{\mathrm{TBS}}$ and the number 


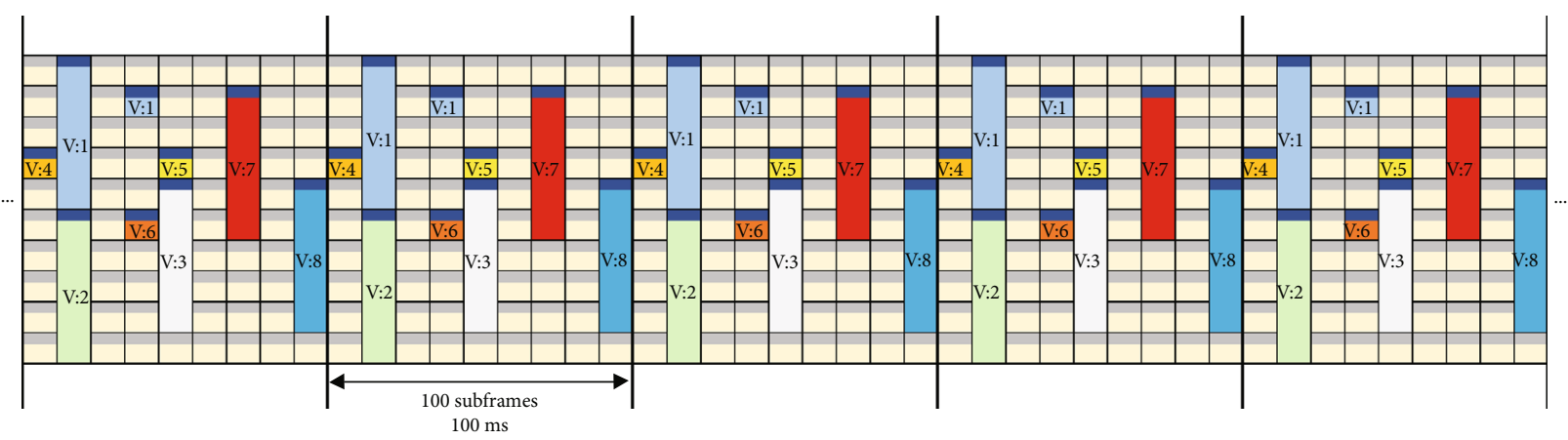

FIgURE 2: The resource grid message allocation keeps repeating periodically over the time (each color represents messages transmitted by different vehicles, and it is also possible that one vehicle sends several messages in one period).

of physical RBs per TB $\left(N_{\mathrm{RB} \_\mathrm{TB}}\right) . N_{\mathrm{CB}}$ is determined by the number of code blocks $C$, being $N_{\mathrm{CB}}=0$ if $C=1$ or $N_{\mathrm{CB}}=$ $C$ if $C>1$, and it is calculated according to section 5.1.2 of [11], which specifies that $N_{\mathrm{CB}}$ should be 0 if messages are smaller than 6144 bits, as it usually is in V2X communications (see Section 4). The number of subchannel bits $\left(N_{\text {BITS_SCH }}\right)$ is calculated considering the 216 bits per RB (for QPSK) or 432 bits per RB (for 16QAM) and then multiplying it by the number of RBs per TB $\left(N_{\mathrm{RB}_{-} \mathrm{TB}}\right)$.

\section{Procedure for PC5 Resource Grid Design}

The administration of any communication system has two clear phases, the design phase and the operation phase. During the system design phase, the problem for the system operator is to choose the correct configuration parameters that maximize the system's capacity. During the operational phase, this problem becomes a scheduling issue as packets of different sizes are required to be transmitted and need to choose an empty Resource Block where to do so, coping also with interference of cochannel transmitters.

In this section, we deal with the first problem and provide a simple mathematical method to choose the grid configuration parameters, which, in this case, are the Resource Block per Subchannel $\left(N_{\mathrm{RB} \_S C H}\right)$, Transport Block Size Index $\left(I_{\mathrm{TBS}}\right)$, and coding rate, that maximize the system's capacity depending on the most common message size.

The first parameter that is required to be stablished is the transmission robustness, in which, according to the 3GPP evaluation methodology [7], the suggested baseline transmission robustness configuration is QPSK with a target coding rate of 0.5 , the optional configuration modes are QPSK with a coding rate of 0.7 and 16QAM with a coding rate of 0.5 , with the first one being the most robust and the last, the least robust. However, 3GPP specifications do not provide transmission robustness in values of coding rate; they use the Transport Block Size Index $\left(I_{\mathrm{TBS}}\right)$. The coding rate can be derived from Table 7.1.7.2.1-1 of [13] which relates, as we said before, the TB size in bits ( $\left.N_{\text {BITS_TB }}\right)$, the TB size in RB $\left(N_{\mathrm{RB} \_\mathrm{TB}}\right)$, and the transmission robustness using the Transport Block Size Index $\left(I_{\mathrm{TBS}}\right)$.

As we will see in Section 4, V2X messages may have different lengths which, for the so-called first day V2X ser- vices, fit in the range between 150 bytes and 350 bytes or 1200 bits and 2800 bits. Table 1 provides the relationship between TB size in RB $\left(N_{\text {RB_TB }}\right)$ and in bits $\left(N_{\text {BITS_TB }}\right)$, $I_{\mathrm{TBS}}$, and coding rate, for the TB sizes that can be used for V2X messages between 1200 bits and 2800 bits, when using QPSK modulation.

As we can see from Table 1, choosing $I_{\mathrm{TBS}}$ with values from 6 to 9 provides the coding rates suggested by the $3 \mathrm{GPP}$ in [7] which range from 0.5 to 0.7 for QPSK modulation.

In order to maximize the system capacity, we have to take into account message transmission distribution. As we will see in Section 4, V2X message generation is quite uniform. Vehicles try to transmit messages notifying their presence at a constant generation rate, and whenever they perceive some unexpected event, they transmit another kind of messages while the event is present. Therefore, the highest percentage of the transmitted messages is the one sent to notify a vehicle's presence, and a good option to optimize the system is to design the resource grid to be able to transmit the maximum amount of these types of messages.

Therefore, once we know the required coding rate and the most common message size, we look up Table 1 to know the appropriate number of RBs per TB $\left(N_{\mathrm{RB}_{-} \mathrm{TB}}\right)$.

The next problem is how we choose the number of RBs per subchannel $\left(N_{\mathrm{RB}_{-} \mathrm{SCH}}\right)$, with the constraints imposed by the $3 \mathrm{GPP}$ (only $5,6,10,15,20,25,50,75$, or $100 \mathrm{RBs}$ per subchannel are possible), that maximizes the system's capacity. Firstly, we define the system's capacity as the number of vehicles that can simultaneously transmit the required V2X messages in the same area, being all of them in coverage (all vehicles are able to receive the messages from all the others) and without overlapping messages of different vehicles in the same RBs, that is, without having collisions. Moreover, with no loss of generality, we can reduce this concept to try to maximize the number of messages that can be sent in a single subframe ( $\left.N_{\text {MSG_SFR }}\right)$, which is the rounded-down integer obtained by

$$
N_{\text {MSG_SFR }}=\left\lfloor\frac{N_{\text {SCH_SFR }}}{N_{\text {SCH_MSG }}}\right\rfloor,
$$


TABLE 1: Relationship between $I_{\mathrm{TBS}}, N_{\mathrm{RB} \_\mathrm{TB}}, N_{\text {BITS_TB }}, N_{\text {BITS_SCH }}$ and the coding rate for QPSK modulation.

\begin{tabular}{|c|c|c|c|c|c|c|c|c|c|}
\hline$I_{\mathrm{TBS}}$ & $N_{\mathrm{RB}_{-} \mathrm{TB}}$ & $N_{\text {BITS_TB }}$ & $N_{\text {BITS_SCH }}$ & Coding rate & $I_{\mathrm{TBS}}$ & $N_{\mathrm{RB}_{-} \mathrm{TB}}$ & $N_{\text {BITS_TB }}$ & $N_{\text {BITS_SCH }}$ & Coding rate \\
\hline 6 & 12 & 1224 & 2592 & 0,48 & 7 & 10 & 1224 & 2160 & 0,58 \\
\hline 6 & 13 & 1352 & 2808 & 0,49 & 7 & 11 & 1302 & 2376 & 0,56 \\
\hline 6 & 14 & 1480 & 3024 & 0,50 & 7 & 12 & 1480 & 2592 & 0,58 \\
\hline 6 & 15 & 1544 & 3240 & 0,48 & 7 & 13 & 1608 & 2808 & 0,58 \\
\hline 6 & 16 & 1672 & 3456 & 0,49 & 7 & 14 & 1672 & 3024 & 0,56 \\
\hline 6 & 17 & 1736 & 3672 & 0,48 & 7 & 15 & 1800 & 3240 & 0,56 \\
\hline 6 & 18 & 1864 & 3888 & 0,49 & 7 & 16 & 1928 & 3456 & 0,56 \\
\hline 6 & 19 & 1992 & 4104 & 0,49 & 7 & 17 & 2088 & 3672 & 0,58 \\
\hline 6 & 20 & 2088 & 4320 & 0,49 & 7 & 18 & 2216 & 3888 & 0,58 \\
\hline 6 & 21 & 2216 & 4536 & 0,49 & 7 & 19 & 2344 & 4104 & 0,58 \\
\hline 6 & 22 & 2208 & 4752 & 0,48 & 7 & 20 & 2472 & 4320 & 0,58 \\
\hline 6 & 23 & 2408 & 4968 & 0,49 & 7 & 21 & 2536 & 4536 & 0,56 \\
\hline 6 & 24 & 2472 & 5184 & 0,48 & 7 & 22 & 2664 & 4752 & 0,57 \\
\hline 6 & 25 & 2600 & 5400 & 0,49 & 7 & 23 & 2792 & 4968 & 0,57 \\
\hline 6 & 26 & 2728 & 5616 & 0,49 & 7 & 24 & 2984 & 5184 & 0,58 \\
\hline 6 & 27 & 2792 & 5832 & 0,48 & & & & 2160 & \\
\hline 6 & 28 & 2984 & 6048 & 0,50 & & & & 2376 & \\
\hline 8 & 9 & 1256 & 1944 & 0,66 & 9 & 8 & 1256 & 1728 & 0,74 \\
\hline 8 & 10 & 1384 & 2160 & 0,65 & 9 & 9 & 1416 & 1944 & 0,74 \\
\hline 8 & 11 & 1544 & 2376 & 0,66 & 9 & 10 & 1544 & 2160 & 0,73 \\
\hline 8 & 12 & 1672 & 2592 & 0,65 & 9 & 11 & 1736 & 2376 & 0,74 \\
\hline 8 & 13 & 1800 & 2808 & 0,65 & 9 & 12 & 1864 & 2592 & 0,73 \\
\hline 8 & 14 & 1928 & 3024 & 0,65 & 9 & 13 & 2024 & 2808 & 0,73 \\
\hline 8 & 15 & 2088 & 3240 & 0,65 & 9 & 14 & 2216 & 3024 & 0,74 \\
\hline 8 & 16 & 2216 & 3456 & 0,65 & 9 & 15 & 2344 & 3240 & 0,73 \\
\hline 8 & 17 & 2344 & 3672 & 0,64 & 9 & 16 & 2536 & 3456 & 0,74 \\
\hline 8 & 18 & 2536 & 3888 & 0,66 & 9 & 17 & 2664 & 3672 & 0,73 \\
\hline 8 & 19 & 2664 & 4104 & 0,65 & 9 & 18 & 2856 & 3888 & 0,74 \\
\hline 8 & 20 & 2792 & 4320 & 0,65 & & & & & \\
\hline 8 & 21 & 2984 & 4536 & 0,66 & & & & & \\
\hline
\end{tabular}

where the number of subchannels per subframe $\left(N_{\mathrm{SCH} \_\mathrm{SFR}}\right)$ is the rounded-down integer computed using

$$
N_{\text {SCH_SFR }}=\left\lfloor\frac{N_{\text {RB_SFR }}}{N_{\text {RB_SCH }}}\right\rfloor \text {, }
$$

with $N_{\text {RB_SFR }}$ being the total number of RBs per subframe (50 RBs per subframe when using a $10 \mathrm{MHz}$ channel bandwidth and $100 \mathrm{RBs}$ for a $20 \mathrm{MHz}$ channel bandwidth).

Additionally, the number of subchannels per message $\left(N_{\text {SCH_MSG }}\right)$ is the rounded-up integer calculated through

$$
N_{\text {SCH_MSG }}=\left\lceil\frac{N_{\text {RB_TB }}+2}{N_{\text {RB_SCH }}}\right\rceil \text {, }
$$

where the number of $\mathrm{RBs}$ per $\mathrm{TB}\left(N_{\mathrm{RB}-\mathrm{TB}}\right)$ is the one obtained from Table 1, and the 2 additional RBs are for SCI information present in the first subchannel of the allocated space.
Consequently, knowing $N_{\mathrm{RB}_{\text {_SFR }}}$ and $N_{\mathrm{RB} \_\mathrm{TB}}$, we have to find out the number of RBs per subchannel ( $\left.N_{\text {RB_SCH }}\right)$ which maximizes the following expression:

$$
N_{\text {MSG_SFR }}=\left\lfloor\frac{\left\lfloor N_{\text {RB_SFR }} / N_{\text {RB_SCH }}\right\rfloor}{\left\lceil\left(N_{R B_{-} \text {TB }}+2\right) / N_{\text {RB_SCH }}\right\rceil}\right\rfloor \text {. }
$$

Due to the fact that this expression has some rounded parts and that the allowed values of $N_{\mathrm{RB}_{3} \mathrm{SCH}}$ are not lineal $(5,6,10,15,20,25,50,75$, or $100 \mathrm{RBs})$, there is no closed solution. To obtain the best $N_{\mathrm{RB} \text { SCH }}$ values, $N_{\mathrm{RB} \text { SCH }} \leq$ $\left(N_{\mathrm{RB} \_\mathrm{TB}}+2\right)$ should be tested and the one that provides the highest $N_{\text {MSG_SFR }}$ should be chosen.

Once the configuration parameters are stablished by the network operator manager, they remain fixed, and the eNB informs UEs of the V2X resource grid structure (including the number of subchannels in a subframe) through the System Information Block (SIB) 21 message transmitted using the Physical Downlink Shared Channel (PDSCH). In-coverage vehicles operating in either Mode 3 
or 4 receive these messages from the LTE network, which may occupy a percentage of the resource grid. However, in this paper, we assume that a separate carrier is used for V2X communications, for example, the $10 \mathrm{MHz}$ unlicensed ITS-G5A carrier at $5.9 \mathrm{GHz}$, while communications with the eNB occur through a traditional licensed LTE carrier. Therefore, the overhead introduced by SIB signalling is not accounted for in our capacity and efficiency calculations.

\section{Analysis of ETSI ITS Protocol Stack Traffic Characteristics}

On top of the wireless access technologies, there are two main protocol stacks for V2X communications, namely, ETSI ITS, used in Europe, and WAVE (Wireless Access in Vehicular Environments), used in the USA [14]. While providing similar functionality, the two stacks differ in the actual messages used. In this paper, we focus on ETSI ITS, although we posit that our conclusions apply also to WAVE.

ETSI ITS defines two main messages for safety-related V2X services, namely, Cooperative Awareness Message (CAM) [15] and Decentralized Environmental Notification Message (DENM) [16]. In the near future, we will have to consider the Collective Perception Messages (CPM), when ETSI finishes the specification. CAMs can be characterized as short messages broadcasted periodically by each vehicle. CAMs from a vehicle inform surrounding vehicles about its status (such as its speed, position, direction, acceleration, curvature, and yaw rate). CAMs are generated at an interval of at least $100 \mathrm{~ms}$ and at most $1000 \mathrm{~ms}$. DENMs and CPMs are event-triggered messages to inform or warn other vehicles about situations such as hazardous road conditions, abnormal traffic situations, or objects detected by onboard sensors. Thus, DENMs and CPMs are transmitted aperiodically unlike CAMs. When using the ETSI ITS protocol stack, these messages are encapsulated in the GeoNetworking (GN) protocol and Basic Transport Protocol (BTP), before being sent to the LTE subchannel.

Protocol header sizes are variable; they have compulsory fields and optional fields. The GeoNetworking header size, although it depends on the target address region, is typically between 40 and 60 bytes. The BTP header has a length of 4 bytes. The typical CAM size falls within the range of 30-300 bytes. The size of a DENM is also variable and is commonly between 60 and 800 bytes. With that, the global size of CAM and DENM messages, which are the most common in V2X communications, falls between 150 bytes and 900 bytes. When it comes to studying their statistical distribution, they do not follow the statistical patterns of Internet traffic, which can be characterized using mathematical models based on exponential, log-normal, Pareto, or Weibull distributions [17]. The reason is that, although there are several optional fields in the V2X message, all of them have a standardized length, so the histogram shape shows that there are several sizes with some probability to be used and other sizes with zero probability. On the contrary, Internet traffic can present any message size; it has a continuous domain from the minimum size when the payload field is zero, to the maximum size defined by the Maximum Transmission Unit (MTU).
There are few studies published about real traffic patterns due to the fact that ITS services are still not operational, and all data comes from field tests performed by some organization or research groups. For instance, in [18], the Car2Car Communication Consortium presents a statistic analysis of ITS-G5 message traces collected in real test drives in Europe in 2018, focused on the CAM messages sent by vehicles of different car makers (Volkswagen, Renault), in different locations, with different ITS-G5 hardware equipment from different vendors. They conclude that CAM sizes can typically be separated into two groups: (i) One group has very small variability, at around 200 bytes, representing around $30 \%$ of the messages. Such messages have no certificates, no pathHistory field. (ii) One group is very diverse, ranging from 300 to 800 bytes, representing around $70 \%$ of the messages. Such messages have certificates and pathHistory variability.

Another CAM message size distribution obtained through a field test can be found in [19]. This case is not as extensive as the previous one but shows a histogram where about $70 \%$ of CAMs have sizes around 190 bytes, about $20 \%$ with sizes close to 320 bytes, and the remaining $10 \%$ have sizes between 350 bytes and 660 bytes. It is also interesting to see that there are no CAMs with sizes between 220 bytes and 300 bytes.

On the other hand, most of the works that require message sizes to study different aspects of the behaviour of V2X communications do not perform field tests and make use of the 3GPP's methodology to evaluate LTE-V2X published in Annex A of [7]. In this document, it is suggested to use "short" CAMs with a size of 190 bytes transmitted 4 times every 5 CAMs, "long" CAMs with a size of 300 bytes transmitted once every 5 CAMs, and DENMs with a size of 800 bytes, all of them including GN and BTP headers. This can be seen, for instance, in $[20,21]$.

In this paper, we present a distribution of real message sizes, whose traces have been captured using a V2X testbed that was developed during years 2017 and 2018 by CELLNEX, i2CAT, and UPC under project V2XArch. In this testbed, four different use cases were developed: Emergency Vehicle Warning (EVW), Cooperative Forward Collision Warning (CFCW), Green Light Optimal Speed Advisory (GLOSA), and multimedia downloading using IPv6 over V2X communications. These applications were implemented on MK5 OBUs and RSUs by Cohda Wireless in that, although they used transmission over IEEE 802.11p, upper layer protocol message sizes are independent of the wireless technology and can be used to empirically determine realistic V2X payload sizes.

Figure 3 shows the distribution of the sizes of CAMs and DENMs (including GN and BTP protocol headers) acquired in the testbeds of the use cases EVW and CFCW. The tests and trials where this data was acquired were performed in two different scenarios. The first one was in an area where CELLNEX has different types of equipment and where we deployed the RSUs. In this scenario, the communication could be direct V2V, or if vehicles were out of sight one from the other, the communication was V-I-V. In this case, RSUs forwarded messages from one area to another using a 


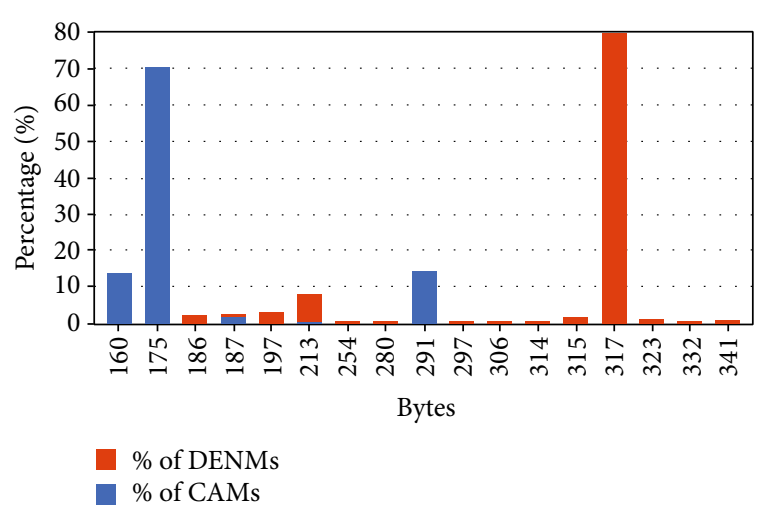

Figure 3: Histogram of CAM and DENM message sizes taken from a COHDA wireless device.

backend server. The other scenario was in mountain roads and highways nearby Barcelona.

In all cases, vehicles always sent 10 CAMs per second. Additionally, in the EVW testbed, DENMs were continuously generated by an ambulance whenever it was moving or stopped. In the CFCW case, DENMs were temporally generated by any vehicle whenever it detected that it was critically approaching another vehicle, in a trajectory of collision. This situation was detected using CAM messages.

We observe that above $80 \%$ of the CAMs transmitted are either 175 bytes or 160 bytes while about $15 \%$ are 291 bytes in size. Meanwhile, about $80 \%$ of DENMs have a 317-byte packet size.

Among the multiple options of message sizes to evaluate the capacity limits in the following chapters, without loss of generality, this paper adopts short CAM message sizes of 190 bytes and long CAM messages sizes of 300 bytes, which are similar to empirical results and the 3GPP proposal, and DENMs of 300 bytes, which are closer to our empirical results.

\section{Results and Discussion about the Capacity Limits of the Adjacent PSCCH-PSSCH PC5 Resource Grid}

The number of CAMs and DENMs that can be transmitted in a given time is constrained by the design of the LTEV2X sidelink resource grid; hence, the number of vehicles that can transmit V2X messages is also bounded. This analysis focuses on the influence that different design parameters of the resource grid have in the final system capacity. For this reason, we do not consider channel reuse that has already been studied in different papers. Our goal is to maximize the number of V2X messages that can be transmitted in a resource grid without considering channel reuse.

In order to design the resource grid, as it has been explained in Section 3, we take the approach to use the most common message size. As per our measurements, it is the 175-byte CAM, and per the suggested evaluation methodology of [7], it is the 190-byte CAM. As both values are very similar, our design will be based on the 190-byte (1520 bits) message.

As a design factor, different possibilities of a subframe division into subchannels, which are able to transmit 1520 bits in the payload using different $I_{\mathrm{TBS}}$, are summarized in Table 2 with data obtained from Table 1 . In the cases where the number of RBs per subframe (50 RBs in a $10 \mathrm{MHz}$ grid) is not divisible by the number of subchannels per subframe, the last subchannel will be bigger than the previous ones in the subframe. If a message does not fully occupy all the RBs of a subchannel, the unused RBs are left empty.

According to the 3GPP evaluation methodology [7], the suggested transmission robustness configuration is QPSK with a target coding rate of 0.5 for the baseline; QPSK with a coding rate of 0.7 , and 16QAM with coding rate of 0.5 are optional, with the first one being the most robust and the last, the least robust.

As per our evaluation, we select the intermediate case in Table 2 where the resource grid is designed to have 6 RBs per subchannel and a robustness defined by $I_{\text {TBS }}$ equal to 9 . With this configuration, it is possible to define 8 subchannels per subframe with each message occupying 2 subchannels each or $10 \mathrm{RBs}$ per $\mathrm{TB}$, which leads to a coding rate of 0.73 . This configuration also provides a $\mathrm{TB}$ size closest to the nominal packet size. Note that the last subchannel of the subframe has $8 \mathrm{RBs}$; therefore, the TB size of the joined last 2 subchannels is $12 \mathrm{RBs}$. This extra space can be used to send the same number of bits with a more robust $I_{\mathrm{TBS}}$ or simply leave it unused.

Now, to allocate "long" CAMs and DENMs, which have a size of 300 bytes, it is possible to either increase the payload size reducing the transmission robustness or maintain the robustness level while increasing the number of RBs per TB $\left(N_{\mathrm{RB} \_\mathrm{TB}}\right)$ by joining more than two subchannels into a bigger one. As we consider that the coverage area of a transmitted message has to be maintained, the approach is to join two or more subchannels into a bigger one. As we have previously defined a basic resource grid structure of 6 RBs per subchannel, we have to continue using this structure. Nevertheless, Table 3 shows two other possible configurations, matching those presented in Table 2.

We thus consider that 300-byte long CAMs and DENMs occupy 4 basic subchannels in the intermediate case that we use per our evaluation. As indicated in Table 3, a 300-byte (2400-bit) long CAM or a DENM fits a 2664-bit TB payload size, defined by a TB formed with $22 \mathrm{RBs}$ coded at QPSK with $I_{\mathrm{TBS}}=7$, which specifies a coding rate of 0.57 . This is slightly more robust than the profile QPSK with coding rate of 0.73 used in short CAMs, which is a good configuration, as information transmitted in DENMs is usually more critical than the one transmitted in CAMs.

In summary, we now have defined the $10 \mathrm{MHz}$ resource grid to be used for the analyses. The resource grid has a total of $50 \mathrm{RBs}$ per subframe, where each subframe is divided into 6 RBs per subchannel. A 190-byte short CAM modulated in QPSK with a code rate of 0.73 occupies two subchannels and uses $I_{\mathrm{TBS}}=9$, while 300-byte long CAMs and DENMs modulated in QPSK with a code rate of 0.57 occupy four subchannels and use $I_{\mathrm{TBS}}=7$. 
TABLE 2: Subchannel design parameters for a $10 \mathrm{MHz}$ grid considering the short CAM (190 bytes $=1520$ bits).

\begin{tabular}{lccccccccc}
\hline $\begin{array}{l}\text { Subchannels } \\
\text { per subframe }\end{array}$ & $\begin{array}{c}\text { RBs per } \\
\text { subchannel } N_{\text {RB_SCH }}\end{array}$ & $\begin{array}{c}\text { Subchannels } \\
\text { per short CAM }\end{array}$ & $\begin{array}{c}\text { Short CAMs } \\
\text { per subframe }\end{array}$ & $\begin{array}{c}\text { RBs per } \\
\text { TB } N_{\text {RB_TB }}\end{array}$ & TB size (bits) & $\begin{array}{c}I_{\text {TBS }} \\
I_{\text {MCS }}\end{array}$ & $\begin{array}{c}\text { Modulation } \\
\text { Coding } \\
\text { rate }\end{array}$ \\
\hline 10 & 5 & 3 & 3 & 13 & 1608 & 7 & 7 & QPSK & 0,58 \\
8 & 6 & 2 & 4 & 10 & 1544 & 9 & 9 & QPSK & 0,73 \\
5 & 10 & 1 & 5 & 8 & 1608 & 11 & 12 & $16 Q A M$ & 0,47 \\
\hline
\end{tabular}

TABLE 3: Subchannel design parameters for a $10 \mathrm{MHz}$ grid considering the long CAM or the DENM (300 bytes $=2400$ bits).

\begin{tabular}{lccccccccc}
\hline $\begin{array}{l}\text { Subchannels } \\
\text { per subframe }\end{array}$ & $\begin{array}{c}\text { RBs per } \\
\text { subchannel } N_{\text {RB_SCH }}\end{array}$ & $\begin{array}{c}\text { Subchannels } \\
\text { per long CAM }\end{array}$ & $\begin{array}{c}\text { Long CAMs } \\
\text { per subframe }\end{array}$ & $\begin{array}{c}\text { RBs per } \\
\text { TB N } N_{\text {RB_TB }}\end{array}$ & TB size (bits) & $\begin{array}{l}I_{\text {TBS }} \\
I_{\text {MCS }}\end{array}$ & $\begin{array}{c}\text { Modulation } \\
\text { Coding } \\
\text { rate }\end{array}$ \\
\hline 10 & 5 & 5 & 2 & 23 & 2408 & 6 & 6 & QPSK & 0,49 \\
5 & 6 & 4 & 2 & 22 & 2664 & 7 & 7 & QPSK & 0,57 \\
5 & 10 & 2 & 2 & 18 & 2536 & 8 & 8 & QPSK & 0,66 \\
\hline
\end{tabular}

As we can see, LTE-V2X regulations enable a high degree of flexibility on defining the resource grid structure which will depend on the choice of each operator. This is a very interesting characteristic, but we also have to understand the impact that this choice has with the traffic model generated by vehicles. To evaluate this aspect, we consider (i) a traffic mix with varying portion of short and large messages, (ii) varying the packet generation interval, and (iii) varying the message sizes.

The target of this evaluation is to compute the maximum number of vehicles that the resource grid can hold under ideal resource scheduling conditions depending on design parameters. Thus, the paper assumes that channel reuse, zoning, collisions, hidden terminal situations, noise, interference, and redundant transmissions are ignored and that as much as possible, the resource grid is fully occupied by messages.

5.1. Capacity with Varying Traffic Mix. In the following two scenarios, the capacity when vehicles generate a mix of CAMs and DENMs is analysed. We consider that $80 \%$ of the CAMs transmitted are short CAMs (190 bytes) while the other $20 \%$ are long CAMs (300 bytes), as used in 3GPP technical reports [7].

We start analysing two scenarios: (i) a case where vehicles generate either CAM or DENM messages every $100 \mathrm{~ms}$ and (ii) a case where vehicles always generate CAMs every $100 \mathrm{~ms}$ and may additionally generate a DENM.

In the scenario shown in Figure 4, a vehicle generates a message $m$ every $100 \mathrm{~ms}$, where the probability of $m$ being a DENM is signalled by the $x$-axis $(x)$, whereas $m$ is a short CAM with the probability equal to $(1-x) * 0.8$ or a long CAM with the probability equal to $(1-x) * 0.2$.

In the next scenario shown in Figure 5, a vehicle generates either only a message $m$, which is a CAM, or a message $m$ together with another message $m^{\prime}$, which is a DENM, every $100 \mathrm{~ms}$. The probability of generating a DENM plus CAM is signalled by the $x$-axis $(x)$. In addition, $m$ is a short CAM with a probability of 0.8 and a long CAM with a probability of 0.2 .

To compute the maximum capacity, we consider the transmission period $t$ that messages generated from a vehicle every $t \mathrm{~ms}$ can be allocated in any subframe within a $t \mathrm{~ms}$ interval.

We can observe from Figures 4 and 5 that, in the best case, when $100 \%$ of vehicles send only a mix of short and long CAMs (without any DENM), the LTE-V2X frame could sustain 334 messages every $100 \mathrm{~ms}$ from 334 vehicles.

On the other hand, when $100 \%$ of vehicles send only DENMs, the LTE-V2X resource grid could sustain up to 200 DENMs every $100 \mathrm{~ms}$, coming from 200 vehicles.

Moreover, when $100 \%$ of vehicles send a DENM together with a CAM, the LTE-V2X frame could sustain only up to 125 CAMs (100 short and 25 long) and 125 DENMs every $100 \mathrm{~ms}$, coming from 125 vehicles.

The observed differences are a consequence of the considered message sizes and, in the second case, the increment of messages per second.

5.2. Capacity with Varying the Transmission Time Interval. The previous scenarios assumed that all message transmission time intervals were $100 \mathrm{~ms}$. We study now the impact of increasing the transmission interval. Figure 6 shows the capacity of the same $10 \mathrm{MHz}$ resource grid with $6 \mathrm{RBs}$ per subchannel and a robustness defined by $I_{\mathrm{TBS}}=9$ for short CAMs (190 bytes) and $I_{\mathrm{TBS}}=7$ for long CAMs and DENMs (300 bytes), when there is a mix of vehicles that transmit a message $m$ every $100 \mathrm{~ms}$ and vehicles that transmit $m$ every $1000 \mathrm{~ms}$. The probability of a vehicle sending $m$ every $100 \mathrm{~ms}$ is indicated by the $x$-axis $(x)$ in Figure 6 , while the probability of a vehicle sending $m$ every $1000 \mathrm{~ms}$ equals $1-$ $x$. In this scenario, $m$ is assumed to be always a short CAM.

When all vehicles transmit only 1 short CAM every $100 \mathrm{~ms}$, the LTE-V2X resource grid can sustain 400 vehicles (as opposed to the 334 vehicles maximum capacity previously discussed, when vehicles transmit an 80-20 percent mix of short and long CAMs). However, when vehicles that transmit every $1000 \mathrm{~ms}$ are introduced into the mix, the capacity of the LTE resource grid increases exponentially, up to a maximum of 4000 messages from unique sources when all of the vehicles transmit every $1000 \mathrm{~ms}$.

As can be observed, an increase in transmission time interval exponentially increases the capacity of the resource 


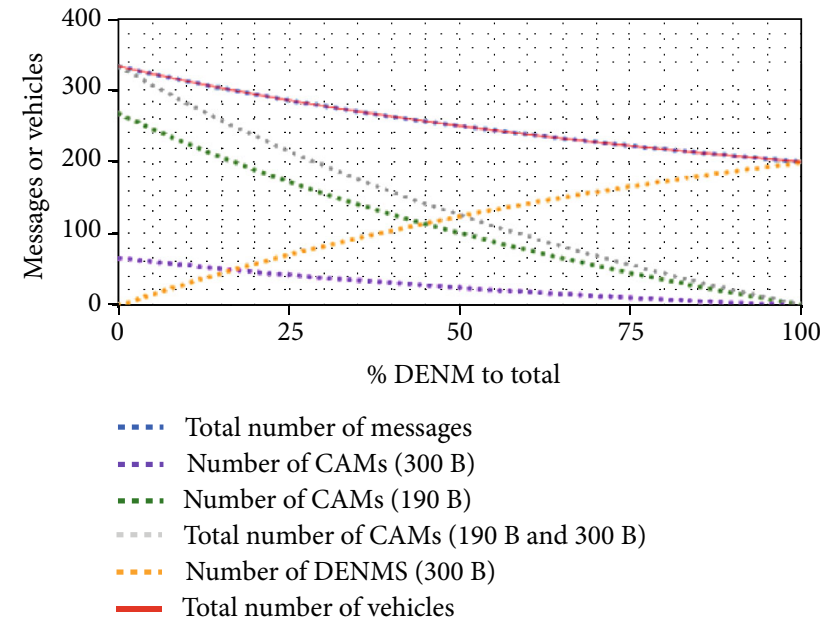

Figure 4: Capacity (number of messages or number of vehicles) with CAM only (190 B and $300 \mathrm{~B})$ or DENM only mix. Transmission period: $100 \mathrm{~ms}$.

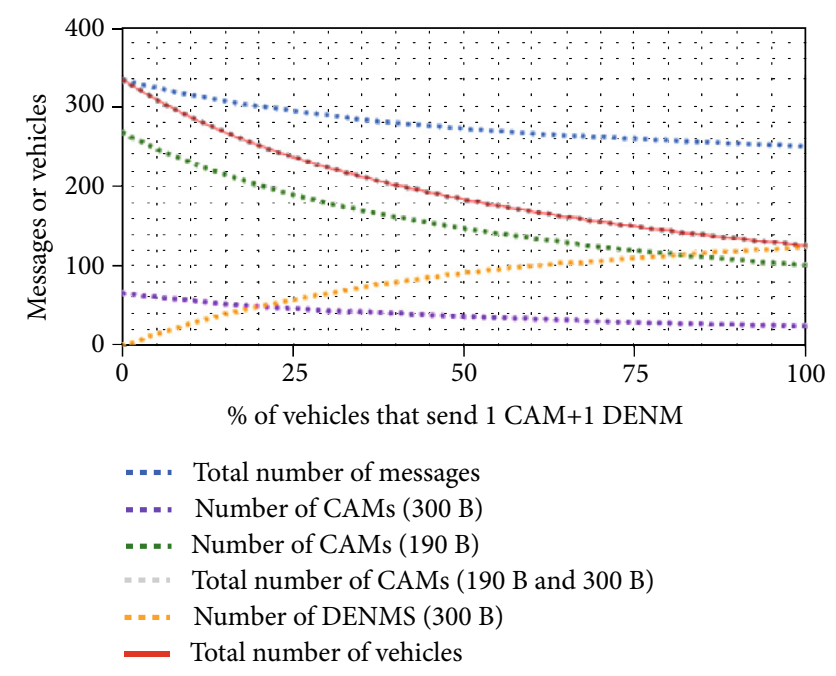

FIGURE 5: Capacity (number of messages or number of vehicles) with CAM only (190 B and $300 \mathrm{~B}$ ) or CAM+DENM. Transmission period: $100 \mathrm{~ms}$.

grid in ideal conditions. Also, varying the transmission time interval could have one of the biggest impacts to capacity, but it is nonlinear. This relationship between transmission time interval and capacity is exploited by IEEE 802.11p, where congestion control is performed by increasing the transmission time interval when there are many vehicles. LTE-V2X would benefit from a similar congestion control mechanism as well.

5.3. Capacity with Varying the Message Size. In the previous scenarios, it was assumed that messages were of fixed length. In the following scenario, the effect of changing the message size itself on the capacity is analysed. The impact of combinations of different parameters such as the Modulation and Coding Scheme (dictated by the $I_{\mathrm{TBS}}$ value) and channel bandwidth (10 MHz vs. $20 \mathrm{MHz}$ ), using $6 \mathrm{RBs}$ per subchan-

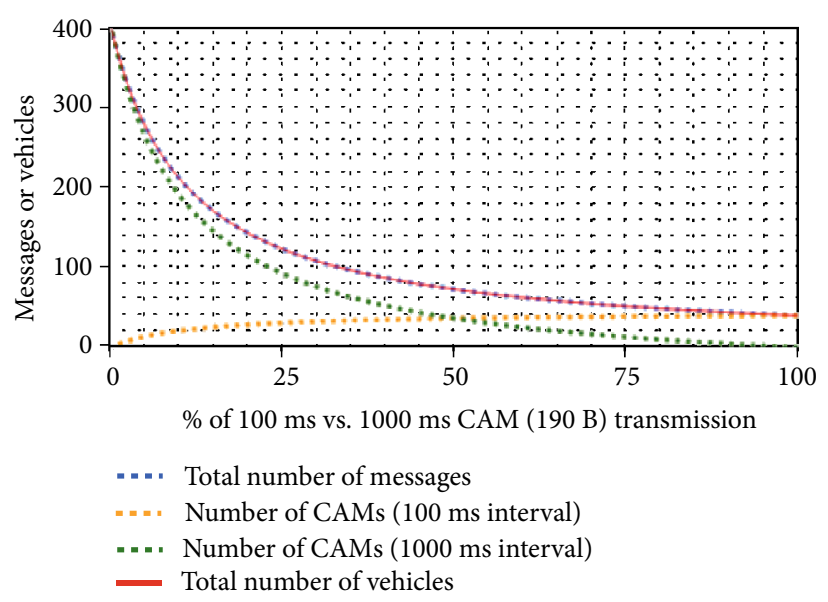

Figure 6: Capacity with a mix of 100 and $1000 \mathrm{~ms}$ transmission periods.

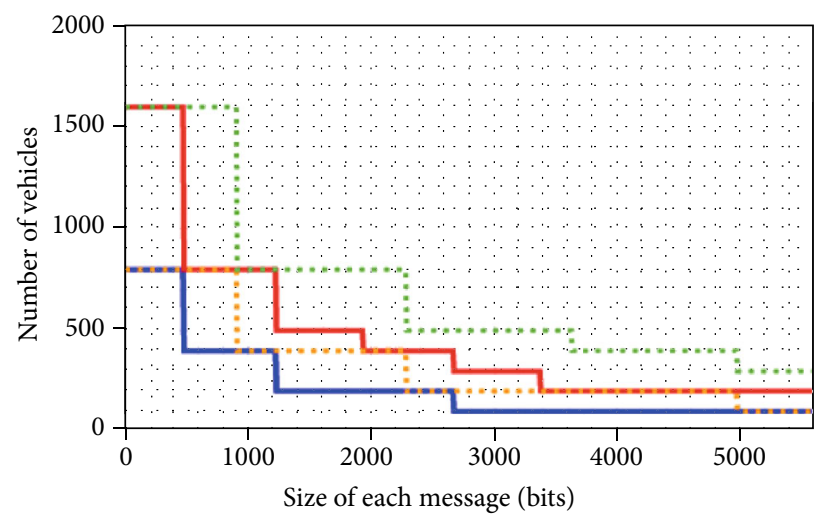

- Number of vehicles for $6 \mathrm{RBs} / \mathrm{SC}$ in $10 \mathrm{MHz}$, $\operatorname{QPSK}\left(I_{\mathrm{TBS}}=7\right)$

- Number of vehicles for $6 \mathrm{RBs} / \mathrm{SC}$ in $20 \mathrm{MHz}$, $\operatorname{QPSK}\left(I_{\mathrm{TBS}}=7\right)$

- = - . Number of vehicles for $6 \mathrm{RBs} / \mathrm{SC}$ in $10 \mathrm{MHz}$, $\operatorname{QPSK}\left(I_{\mathrm{TBS}}=12\right)$

.... Number of vehicles for $6 \mathrm{RBs} / \mathrm{SC}$ in $20 \mathrm{MHz}$, QPSK $\left(I_{\text {TBS }}=12\right)$

FIGURE 7: Capacity when message size is varied.

nel, is considered. The target coding rate for all transmissions is 0.5 ; thus, we compare $I_{\mathrm{TBS}}=7$ (QPSK) vs. $I_{\mathrm{TBS}}=12$ (16QAM). It is assumed that the messages are transmitted at a period of $100 \mathrm{~ms}$. Ideal resource scheduling conditions are again assumed. Figure 7 illustrates this scenario.

As depicted in Figure 7, the number of messages sustained by the LTE frame decreases in a staircase-like manner as the message size increases. The reason behind this can be visualized better in Figure 8, which focuses on the case with a $10 \mathrm{MHz}$ resource grid with a division of $6 \mathrm{RBs}$ per subchannel and QPSK $\left(I_{\mathrm{TBS}}=7\right)$ (blue line in Figure 7$)$. Thus, in Figure 8(a), 8 messages of up to 472 bits, occupying one subchannel each, have TBs that are partially occupied by the useful data of the message, 2 RBs occupied by the SCI, and the rest unused space. This corresponds to a resource grid message capacity of 800 messages given a transmission time 


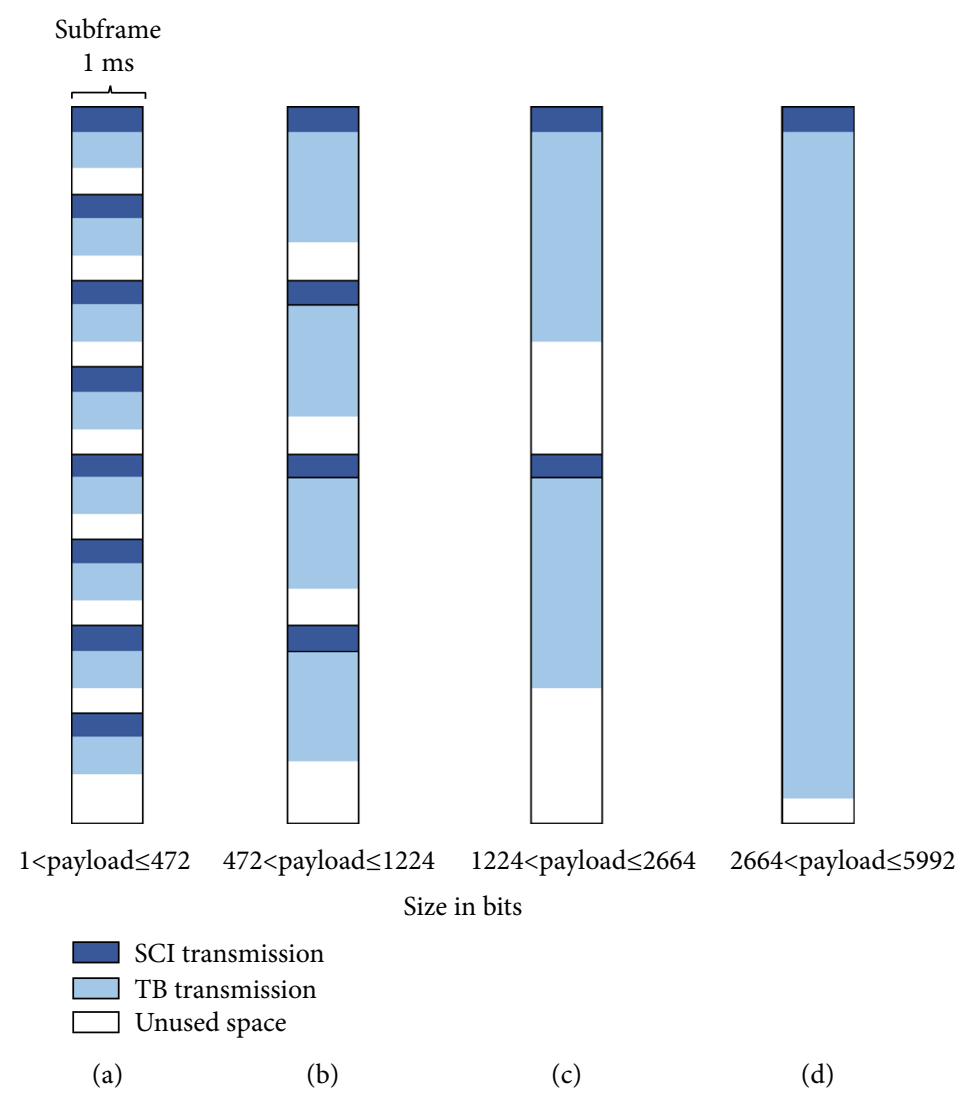

FIGURE 8: Effect of growing message size on subchannel occupancy and subframe capacity for $6 \mathrm{RBs} / \mathrm{SC}$ in $10 \mathrm{MHz}, \mathrm{QPSK}\left(I_{\mathrm{TBS}}=7\right)$. Blue line of Figure 7.

interval of $100 \mathrm{~ms}$. As the message size is increased, the unused space is gradually filled up, until all the unused space is occupied when the message has exactly 472 bits of payload. However, in Figure $8(\mathrm{~b})$, when the message size is increased further, up to 1224 bits of payload, one subchannel is no longer sufficient to accommodate the message. Thus, an additional subchannel has to be allocated per message, since the smallest unit of resource allocation in LTE$\mathrm{V} 2 \mathrm{X}$ is in subchannels rather than in RBs. An additional subchannel per message means that the subframe can now be filled by only 4 messages of the same size instead of 8 , resulting in a capacity drop from 800 to 400 vehicles. Resource grid capacity in LTE-V2X is thus extremely sensitive to the message size.

As the message size is increased further, the newly available unused space from the extra subchannel is gradually filled up until all the space for data is fully. The process continues until only one message fully occupies an entire subframe for all subframes in the resource grid (Figure 8(d)). In this case, the resource grid message capacity is 100 messages. Returning to Figure 7, it can be observed that increasing the bandwidth from $10 \mathrm{MHz}$ to $20 \mathrm{MHz}$ increases the message capacity by a factor of 2 , while increasing the robustness of the modulation changing $I_{\mathrm{TBS}}$ from 7 to 12 means that each subchannel could carry approximately twice as much information; thus, the steps are about twice as wide. However, they are not exactly double due to the fact that coding rate steps are nonlinear.

\section{Results and Discussion about the Efficiency Limits in the LTE-V2X Resource Grid}

The efficiency of the resource grid is affected and decreased by three factors: the coding rate described in Section 5 , the two RBs that are automatically dedicated for control in each message transmission, and, depending on the size of the subchannels and the size of the message, the unused space on the TB. In this section, the efficiency in the LTE-V2X resource grid is investigated.

Efficiency is defined here simply as the ratio of the size taken up by the sum of all upper-layer message bits over the total size occupied (in the case of QPSK and $10 \mathrm{MHz}$ bandwidth, 216 bits per RB by $50 \mathrm{RBs).} \mathrm{In} \mathrm{the} \mathrm{following} \mathrm{sce-}$ nario, again a channel bandwidth of $10 \mathrm{MHz}$ is used; thus, there would be $50 \mathrm{RBs}$ per subframe. It is assumed that the Transport Block Size Index is $I_{\mathrm{TBS}}=7$ (QPSK), looking for a target coding rate for all transmissions close to 0.5 . All messages are assumed to be of the same size and are transmitted at a period of $100 \mathrm{~ms}$. Also, ideal resource scheduling conditions are again assumed, and zoning and redundant transmissions are ignored.

The efficiency as the message size which is increased using resource grids with $5,6,10$, and $25 \mathrm{RBs}$ per subchannel is illustrated in Figure 9. The resulting sawtooth-like shape is due to the jumps in the number of messages per subframe. This is the same reason for the staircase-like shape of the capacity graph in the scenario in Section 5.3. 


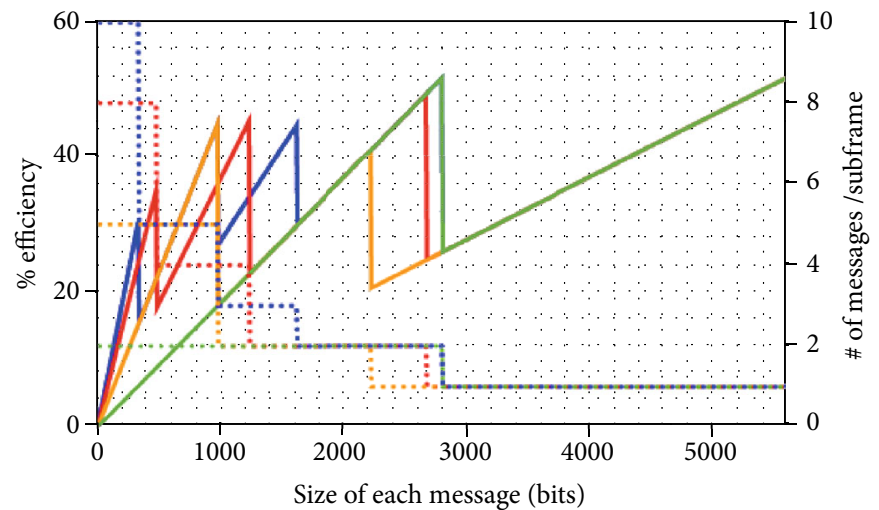

$$
\begin{aligned}
& \text { - Efficiency for } 5 \mathrm{RBs} / \mathrm{SC} \\
& \text { Efficiency for } 6 \mathrm{RBs} / \mathrm{SC} \\
& \text { Efficiency for } 10 \mathrm{RBs} / \mathrm{SC} \\
& \text { - - - - Mesficiency for } 25 \mathrm{RBs} / \mathrm{SC} \\
& \text { - - - - Messages per subframe for } 5 \mathrm{RBs} / \mathrm{SC} \\
& \text { - - - - Messages per subframe for } 10 \mathrm{RBs} / \mathrm{SC} \\
& \text { - - - Messages per subframe for } 25 \mathrm{RBs} / \mathrm{SC}
\end{aligned}
$$

FIgURE 9: Message size vs. efficiency for $I_{\mathrm{TBS}}=7$ (QPSK) at $10 \mathrm{MHz}$ channelization.

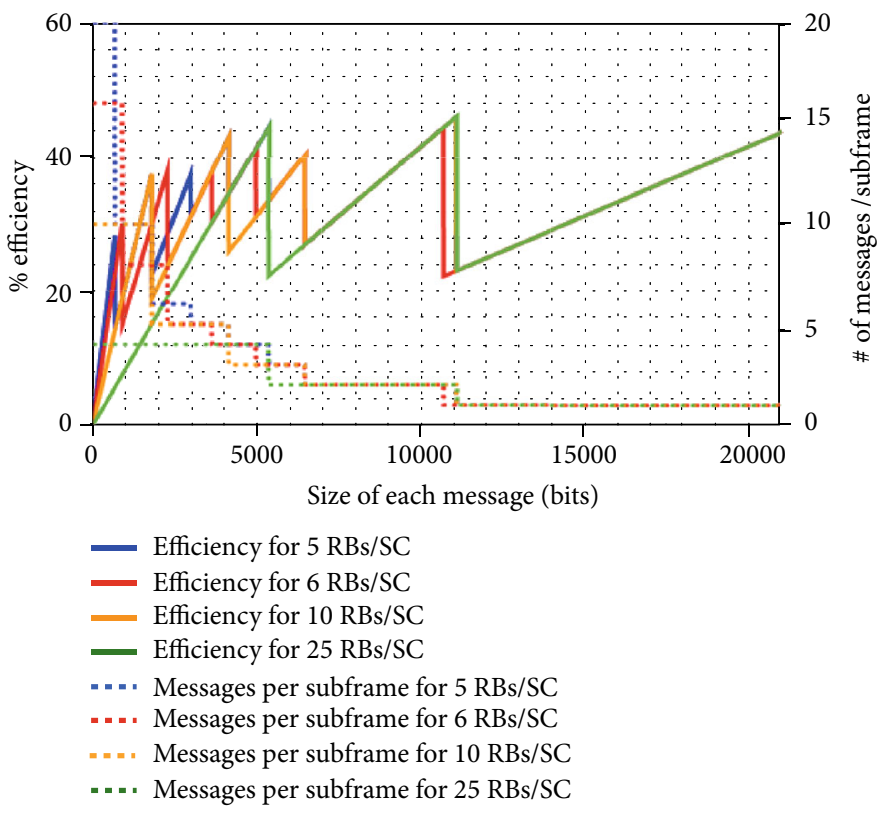

FIgURE 10: Message size vs. efficiency for $I_{\mathrm{TBS}}=12$ (16QAM) at $20 \mathrm{MHz}$ channelization.

In Figure 9, peaks are due to the local maxima of efficiency; i.e., the message perfectly fills all the allocated space inside a TB (as in subframe 3 of Figure 8). However, when being in this situation and the message size increases even by a single bit, the current TB size is not sufficient and an additional subchannel needs to be allocated to the TB. The new TB will only be partially filled (as in subframe 4 of Figure 8). This extra unoccupied space of the TB, appearing just when message sizes overpass certain size thresholds, causes sudden drops in efficiency.

It can be observed that the local peak efficiencies generally increase with larger message sizes. This is because larger message sizes mean that there are fewer messages on the resource grid; consequently, the overhead taken up by the SCIs diminishes. For instance, in Figure 8, there are only 4 messages in subframe 8 compared to subframe 3 which has 8 messages, but both subframes are fully occupied. However, subframe 8 is more efficient in that there are fewer RBs occupied by SCIs and more useful data in subframe 8 compared to subframe 3 . This means that there is a trade-off between efficiency and number of messages that could be accommodated: having larger messages generally increases efficiency because more space is spent on transmitting useful data rather than control information; however, 
this means decreasing the number of messages that the resource grid can accommodate. With a target coding rate of 0.5 , the maximum efficiency that could be achieved is around 50\%. However, even at the largest possible message size, the efficiency would be under the percentage imposed by the coding rate because of the presence of the SCIs. Upon a closer look at Figure 9, it could be seen that it is not always the case that the local peaks in efficiency are monotonically increasing with message size. This could be explained in subframe 9 of Figure 8. Subframe 9 has 2 messages each occupying 3 subchannels, but it also has 2 remaining subchannels that cannot be occupied by a message of the same size. This contributes to significant inefficiency.

The efficiency on a $20 \mathrm{MHz}$ resource grid using $I_{\mathrm{TBS}}=12$ (16QAM), also targeting a coding rate of 0.5 , is shown on Figure 10. The general trend is similar to that of the previous scenario, except that each subchannel has twice the capacity due to the increase in modulation and that twice as many messages can be accommodated due to the increase from 10 to $20 \mathrm{MHz}$; thus, the capacity is increased fourfold.

\section{Conclusions}

In this paper, we have investigated the effect of resource grid design parameters in the capacity and efficiency of LTE-V2X as it will be used to transmit different types of data in vehicular networks, from position or speed of the vehicle up to much of its sensor data.

Our main contribution is to expose the sensitivity of LTE-V2X performance parameters to the three basic resource grid design parameters (Resource Block per Subchannel, Transport Block Size Index, and coding rate) and also to the traffic pattern generated by vehicles.

In order to present these results, we do not consider channel reuse and radio propagation conditions that have already been studied by other authors. Nevertheless, as channel reuse improves LTE-V2X global capacity in a geographical region, the values presented in this paper have to be considered as the upper bound of correctly received messages possibly received by a specific vehicle.

Our main findings are that capacity and efficiency in LTE-V2X are very sensitive to the relation between message size and subchannel configuration. For instance, the number of messages sustained by the LTE-V2X resource grid decreases in a staircase-like manner as the message size increases; therefore, a single increase of one byte in the message size could produce a reduction close to $50 \%$ on the number of messages that can fit in this resource grid.

An implication of this high sensitivity is that mobile network operators would have to deeply study the traffic pattern of vehicles in their area of service in order to define LTE-V2X operating parameters.

\section{Data Availability}

The only data acquired from real test beds is that of Figure 3 and it will be available from the corresponding author upon request.

\section{Conflicts of Interest}

The authors declare no conflict of interest.

\section{Acknowledgments}

This work has been supported in part by the ERDF and the Spanish Government through projects TEC2016-79988-P and TEC2016-76795-C6-2-R; by the Spanish Ministerio de Industria, Energía y Turismo and CELLNEX through project TSI-100102-2015-13 V2XArch; and by the ERDF and Secretaria d'Universitats i Recerca de la Generalitat de Catalunya through projects 2017-SGR-00376 and FEM IoT 001-P001662. Additionally, part of this work has been produced in the master's thesis of Leandro Miguel Lopez [22] that he carried out at the Universitat Politècnica de Catalunya.

\section{References}

[1] O. Kaiwartya, A. H. Abdullah, Y. Cao, A. Altameem, and M. Prasad, "Internet of vehicles: motivation, layered architecture, network model, challenges, and future aspects," IEEE Access, vol. 4, pp. 5356-5373, 2016.

[2] A. Bazzi, B. M. Masini, A. Zanella, and I. Thibault, "On the performance of IEEE $802.11 \mathrm{p}$ and LTE-V2V for the cooperative awareness of connected vehicles," IEEE Transactions on Vehicular Technology, vol. 66, no. 11, pp. 10419-10432, 2017.

[3] R. Molina-Masegosa and J. Gozalvez, "System level evaluation of LTE-V2V mode 4 communications and its distributed scheduling," in 2017 IEEE 85th Vehicular Technology Conference (VTC Spring), Sydney, Australia, 2017.

[4] R. Molina-Masegosa and J. Gozalvez, "LTE-V for sidelink 5G V2X vehicular Communications: A New 5G Technology for Short-Range Vehicle-to-Everything communications," IEEE Vehicular Technology Magazine, vol. 12, no. 4, pp. 30-39, 2017.

[5] A. Bazzi, G. Cecchini, B. M. Masini, and A. Zanella, "Study of the impact of PHY and MAC parameters in 3GPP C-V2V mode 4," IEEE Access, vol. 6, pp. 71685-71698, 2018.

[6] K. N. Qureshi, A. H. Abdullah, O. Kaiwartya, S. Iqbal, R. A. Butt, and F. Bashir, "A dynamic congestion control scheme for safety applications in vehicular ad hoc networks," Computers \& Electrical Engineering, vol. 72, pp. 774-788, 2018.

[7] 3rd Generation Partnership Project (3GPP), Technical Specifcation Group Radio Access Network. Study on LTE-based V2X Services (Release 14), 3GPP, 2016, TR 36.885 V14.0.0.0.

[8] Y. Park, S. Weon, I. Hwang, H. Lee, J. Kim, and D. Hong, "Spatial capacity of LTE-based V2V communication," in 2018 International Conference on Electronics, Information, and Communication (ICEIC), Honolulu, USA, 2018.

[9] A. Bazzi, B. M. Masini, and A. Zanella, "How many vehicles in the LTE-V2V awareness range with half or full duplex radios?," in 2017 15th International Conference on ITS Telecommunications (ITST), Warsaw, Poland, 2017.

[10] 3rd Generation Partnership Project (3GPP), Evolved Universal Terrestrial Radio Access (E-UTRA); User Equipment (UE) Radio Transmission and Reception (Release 15), 3GPP, 2019, TS 36.101 V15.8.0.

[11] 3rd Generation Partnership Project (3GPP), Evolved Universal Terrestrial Radio Access (E-UTRA); Multiplexing and Channel Coding (Release 15), 3GPP, 2019, TS 36.212 V15.7.0. 
[12] 3rd Generation Partnership Project (3GPP), Evolved Universal Terrestrial Radio Access (E-UTRA); Radio Resource Control (RRC); Protocol Specification (Release 15), 3GPP, 2019, TS 36.331 V15.7.0.

[13] 3rd Generation Partnership Project (3GPP), Evolved Universal Terrestrial Radio Access (E-UTRA); Physical Layer Procedures (Release 15), 3GPP, 2019, TR 36.213 V15.7.0.

[14] IEEE, IEEE standard for Wireless Access in Vehicular Environments (WAVE) - Networking Services, IEEE, 2016, Std 1609.32016.

[15] European Telecommunications Standards Institute (ETSI), Intelligent Transport Systems (ITS); Vehicular Communications; Basic Set of Applications; Part 2: Specification of Cooperative Awareness Basic Service, ETSI, 2019, TS 102 637-2 V1.4.1.

[16] European Telecommunications Standards Institute (ETSI), Intelligent Transport Systems (ITS); Vehicular Communications; Basic Set of Applications; Part 3: Specifications of Decentralized Environmental Notification Basic Service, ETSI, 2019, TS 102 637-3 V1.3.1.

[17] E. R. S. Castro, M. S. Alencar, and I. E. Fonseca, "Probability density functions of the packet length for computer networks with bimodal traffic," International Journal of Computer Networks \& Communications, vol. 5, no. 3, pp. 17-31, 2013.

[18] V. Martinez and F. Berens, Survey on ITS-G5 CAM Statistics. C2CC_TR_2052, version 1.0.1, Car2Car Communication Consortium, 2018.

[19] V. Mannoni, V. Berg, S. Sesia, and E. Perraud, "A comparison of the V2X communication systems: ITS-G5 and C-V2X," in 2019 IEEE 89th Vehicular Technology Conference (VTC2019Spring), Kuala-Lumpur, Malaysia, 2019.

[20] A. Bazzi, G. Cecchini, M. Menarini, B. M. Masini, and A. Zanella, "Survey and perspectives of vehicular Wi-Fi versus sidelink cellular-V2X in the $5 \mathrm{G}$ era," Future Internet, vol. 11, no. 6, p. 122, 2019.

[21] M. Gonzalez-Martín, M. Sepulcre, R. Molina-Masegosa, and J. Gozlavez, "Analytical models of the performance of C-V2X mode 4 vehicular communications," IEEE Transactions on Vehicular Technology, vol. 68, no. 2, pp. 1155-1166, 2019.

[22] L. M. W. Lopez, Performance of sensing-based semi-persistent scheduling (SPS) in LTE-V2X release 14 distributed mode, [M.S. thesis], Universitat Politècnica de Catalunya, 2019. 